\title{
Improved classical limit analogues for Galton-Watson processes with or without immigration
}

\author{
C.C. Heyde and J.R. Leslie
}

\begin{abstract}
It has recently emerged that the central limit theorem and iterated logarithm law for random walk processes have natural counterparts for Galton-Watson processes with or without imigration. Much of the work on these counterparts has previously involved the imposition of supplementary moment conditions. In this paper we show how to dispense with these supplementary conditions and in so doing make the analogy with the random walk results complete.
\end{abstract}

\section{Introduction}

Let $Z_{0}=1, Z_{1}, Z_{2}, \ldots$ denote a super-critical Galton-Watson process with $1<E Z_{1}=m$ and $0<\operatorname{var} Z_{1}=\sigma^{2}<\infty$. It is well-known that there exists a non-degenerate random variable $W$ such that $\lim _{n \rightarrow \infty} W_{n}=W$

almost surely, where $w_{n}=m^{-n} z_{n}$ (for example, Harris [2], p. 13).

Furthermore, some central limit analogues have been established in this context by Heyde [5] and Bühler [1]. These results are that $\left(m^{2}-m\right)^{\frac{1}{2}} \sigma^{-1} z_{n}^{-\frac{1}{2}} m^{n}\left(W-W_{n}\right)$ conditional on $z_{n}>0$ and $\left(m^{2}-m\right)^{\frac{1}{2} \sigma^{-1}} m^{-\frac{1}{2} j}\left(m^{j}-1\right)^{-\frac{1}{2}} Z_{n}^{-\frac{1}{2}}\left(z_{r_{l}+j^{-n}} z_{n}\right)$ conditional on $z_{n}>0$ (fixed $j$ ) are both asymptotically normal $N(0,1)$. See [5] for an explanation of 
why these results can be regarded as central limit analogues.

Under the further restriction that $E Z_{1}^{3}<\infty$, rates of convergence in the central limit analogues cited above have been given in [7]. These have been used in [6] to obtain almost sure convergence results for the Galton-Watson process which are analogues of the law of the iterated logarithm for random walks. In this paper we shall show how the restriction that $E Z_{1}^{3}<\infty$ may be removed. In Section 2 we shall obtain convergence rates in the central limit analogues and also iterated logarithm analogues under the basic condition that $E Z_{1}^{2}<\infty$.

In Section 3 of this paper we shall deal with the Galton-Watson process with immigration. The development of the corresponding limit results in this case has followed the pattern described previously for the case without immigration. Heyde and Seneta [8] have obtained central limit analogues under $E Z_{1}^{2}<\infty$ and rate results and iterated logarithm analogues under $E Z_{1}^{3}<\infty$. We shall again show how to dispense with the moment restriction and will obtain the rate results and iterated logarithm analogues under $E Z_{1}^{2}<\infty$.

\section{The process without immigration}

We shall establish the following theorems. The reader is referred to the papers [5], [6] and [7] for background details.

THEOREM 1. Let $1<m=E Z_{1}$ and $0<\operatorname{var} Z_{1}=\sigma^{2}<\infty$. Then

$$
\sup _{x}\left|P\left(\left(m^{2}-m\right)^{\frac{1}{2}} \sigma^{-1} u_{n}^{-1} z_{n}^{-\frac{1}{2}} m^{n}\left(W-W_{n}\right) \leq x \mid z_{n}>0\right)-\Phi(x)\right| \leq c_{n}
$$

and

$$
\sup _{x}\left|P\left(\sigma_{r}^{-1} v_{z}^{-1} z_{n}^{-\frac{1}{2}}\left(z_{n+p^{-m}} z_{n}\right) \leq x \mid z_{n}>0\right)-\Phi(x)\right| \leq d_{n},
$$

where $\left\{c_{n}\right\},\left\{d_{n}\right\}$ are certain sequences of positive constants satisfying $\sum_{n=1}^{\infty} c_{n}<\infty$ and $\sum_{n=1}^{\infty} d_{n}<\infty$. Here

$$
\sigma_{r}^{2}=\operatorname{var} Z_{r}=\sigma^{2} m^{r}\left(m^{r}-1\right)\left(m^{2}-m\right)^{-1},
$$


$r$ any fixed integer,

$$
\begin{aligned}
& u_{n}=\int_{|x|<\sqrt{n}} x^{2} d P\left(\sigma^{-1}\left(m^{2}-m\right)^{\frac{1}{2}}(W-1) \leq x\right) \\
& v_{n}=\int_{|x|<\sqrt{n}} x^{2} d P\left(\sigma_{r}^{-1}\left(z_{r}-m^{r}\right) \leq x\right)
\end{aligned}
$$

and $\Phi(x)$ is the distribution function of $N(0,1)$.

Explicit forms for $c_{n}$ and $d_{n}$ can be found by applying the lemma below. We note also that $u_{n} \uparrow 1$ and $v_{n} \uparrow 1$ as $n \rightarrow \infty$.

THEOREM 2. Suppose that $1<m=E Z_{1}$ and $0<\operatorname{var} Z_{1}=\sigma^{2}<\infty$. Then, on the non-extinction set $\{W>0\}$ we have almost surely

$$
\lim _{n \rightarrow \infty} \frac{z_{n+r^{-m}} z_{n}}{\left(2 \sigma_{r}^{2} z_{n} \log n\right)^{\frac{1}{2}}}=1, \quad \lim _{n \rightarrow \infty} \inf \frac{z_{n+r^{-m^{r}} z_{n}}}{\left(2 \sigma_{r}^{2} z_{n} \log n\right)^{\frac{1}{2}}}=-1
$$

and

$\lim _{n \rightarrow \infty} \frac{m^{n} W-Z n}{\left(2 \sigma^{2}\left(m^{2}-m\right)^{-1} z_{n} \log n\right)^{\frac{1}{2}}}=1, \quad \lim _{n \rightarrow \infty} \inf \frac{m^{n} W-Z n}{\left(2 \sigma^{2}\left(m^{2}-m\right)^{-1} z_{n} \log n\right)^{\frac{1}{2}}}=-1$,

where $r$ is any fixed positive integer.

Theorems 1 and 2 extend the scope of results given in Heyde and Brown [7] and in Heyde [6] respectively under the additional condition that $E Z_{1}^{3}<\infty$. The form of the bounds obtained in Theorem 1 is however, of necessity, much more complicated in the general case. An explanation of this is not difficult to deduce from results given in [3] and [4]. Our Theorem 2 preserves exactly the form of the Theorem of [6] under the more general conditions.

In order to establish the above results we need the following key lemma. The result of the lemma is given in two parts; the first is needed in the present section and the second to obtain corresponding results for the process with immigration in Section 3.

LEMMA. Let $\xi_{i}, i=1,2,3, \ldots$ be independent and identically 
distributed random variables with $E\left(\xi_{1}\right)=0$ and $\operatorname{var} \xi_{1}=\alpha^{2}<\infty$. Let $N_{n}$ be a positive integer valued random variable which is independent of the $\left\{\xi_{i}\right\}$. Then,

(1) $\sup _{x}\left|P\left(\alpha^{-1} d_{n}^{-1} N_{n}^{-\frac{1}{2}}\left(\xi_{1}+\ldots+\xi_{N_{n}}\right) \leq x\right)-\Phi(x)\right|$

$$
\leq A E\left(N^{-\frac{1}{2}} a_{N}\right)+B E\left(N_{n}^{\frac{1}{2}} b_{N}\right)+E\left(N_{n} c_{N_{n}}\right)
$$

where $A, B$ are positive constants and

$$
\begin{array}{ll}
a_{n}=\int_{|x|<\sqrt{n}}|x|^{3} d P\left(\alpha^{-1} \xi_{1} \leq x\right), & b_{n}=\int_{|x| \geq \sqrt{n}}|x| d P\left(\alpha^{-1} \xi_{1} \leq x\right), \\
c_{n}=P\left(\alpha^{-1}\left|\xi_{I}\right|>\sqrt{n}\right), & d_{n}^{2}=\int_{|x|<\sqrt{n}} x^{2} d P\left(\alpha^{-1} \xi_{I} \leq x\right) .
\end{array}
$$

If $\eta_{n}$ with $E\left|\eta_{n}\right|<\infty$ is a random variable which is independent of the $\left\{\xi_{i}\right\}$ and of $N_{n}$, then for any sequence $\left\{\varepsilon_{n}\right\}$ of positive constants with $\varepsilon_{n} \rightarrow 0$ as $n \rightarrow \infty$,

$$
\begin{aligned}
& \sup _{x}\left|P\left(\alpha^{-1} \bar{d}_{N}^{-1} N_{n}^{-\frac{1}{2}}\left(\xi_{I}+\ldots+\xi_{N_{n}}+\eta_{n}\right) \leq x\right)-\Phi(x)\right| \\
& \quad \leq A E\left(N_{n}^{-\frac{1}{2}} a_{N_{n}}\right)+B E\left(N_{n}^{\frac{1}{2}} b_{N}\right)+E\left(N_{n} c_{N_{n}}\right)+\alpha^{-1} \varepsilon_{n}^{-1} E\left|\eta_{n}\right| E\left(N_{n}^{-\frac{1}{2}} d_{N}^{-1}\right)+\frac{1}{2} \varepsilon_{n} .
\end{aligned}
$$

Proof. Let

$$
e_{n}^{2}=\int_{|x|<\sqrt{n}} x^{2} d P\left(\alpha^{-1} \xi_{1} \leq x\right)-\left(\int_{|x|<\sqrt{n}} x d P\left(\alpha^{-1} \xi_{1} \leq x\right)\right)^{2} .
$$

We have

$$
\begin{array}{r}
\sup _{x}\left|P\left(\alpha^{-1} d_{N}^{-1} N_{n}^{-\frac{1}{2}}\left(\xi_{1}+\ldots+\xi_{N_{n}}\right) \leq x \mid N_{n}=k\right)-\Phi(x)\right| \\
\quad \leq \sup _{x}\left|P\left(\alpha^{-1} e_{N_{n} N_{n}^{-1}}^{-\frac{1}{2}}\left(\xi_{1}+\ldots+\xi_{N_{n}}\right) \leq x \mid N_{n}=k\right)-\Phi(x)\right| \\
+\sup _{x}\left|\Phi(x)-\Phi\left(e_{k} d_{k}^{-1} x\right)\right| .
\end{array}
$$

Also, using the mean value theorem, 
(4) $\sup _{x}\left|\Phi(x)-\Phi\left(e_{k} d_{k}^{-1} x\right)\right| \leq c\left(1-e_{k} d_{k}^{-1}\right)$

$$
\begin{aligned}
& =c d_{k}^{-1}\left(e_{k}+d_{k}\right)^{-1}\left(\int_{|x|<\sqrt{k}} x d P\left(\alpha^{-1} \xi_{1} \leq x\right)\right)^{2} \\
& =c d_{k}^{-1}\left(e_{k}+d_{k}\right)^{-1}\left(\int_{|x| \geq \sqrt{k}} x d P\left(\alpha^{-1} \xi_{1} \leq x\right)\right)^{2} \\
& \leq c_{1} b_{k}^{2} \leq c_{1} b_{k},
\end{aligned}
$$

$c, c_{1}$ being positive constants. Furthermore, from (22) of Heyde [4] we find that

$$
\text { (5) } \begin{aligned}
\sup _{x} \mid P\left(\alpha^{-1} e_{N}^{-1} N_{n}^{-\frac{1}{2}}\left(\xi_{1}+\ldots+\xi_{N}\right) \leq x \mid N_{n}=k\right) & -\Phi(x) \mid \\
& \leq A k^{-\frac{1}{2}} a_{k}+B_{1} k^{\frac{1}{2} b_{k}}+k c_{k},
\end{aligned}
$$

so that using (3), (4) and (5),

$$
\text { (6) } \begin{aligned}
\sup _{x} \mid P\left(\alpha^{-1} d_{N}^{-1} N_{n}^{-\frac{1}{2}}\left(\xi_{I}+\ldots+\xi_{N_{n}}\right) \leq x \mid N_{n}=k\right) & -\Phi(x) \mid \\
& \leq A k^{-\frac{1}{2}} a_{k}+B k^{\frac{1}{2} b_{k}}+k c_{k} .
\end{aligned}
$$

The result (1) follows readily from (6) using the argument of the lemm in $\$ 4$ of Heyde and Brown [7]. (2) is obtained using exactly the method of Lemma 2.1 of Heyde and Seneta [8] with the aid of (1) instead of the Berry-Esseen bound.

Proof of Theorem 1. Suppose that $2_{n}^{*}$ has the distribution of $z_{n}$ conditional on $z_{n}>0$. We firstly note that (see [5], [7]), conditional on $z_{n}>0, m^{n} z_{n}^{-\frac{1}{2}}\left(W-W_{n}\right)$ has the same distribution as $\left(z_{n}^{*}\right)^{-\frac{1}{2}}\left(U_{1}+\ldots+U_{Z_{n}^{*}}\right)$, where the $U_{i}$ are independent of $z_{n}^{*}$ and are independent and identically distributed, each with the distribution of $w-1$. Also, conditional on $z_{n}>0, z_{n}^{-\frac{1}{2}}\left(z_{n+x^{-}}-m_{n}^{r} z_{n}\right)$ has the same distribution as $\left(z_{n}^{*}\right)^{-\frac{1}{2}}\left(v_{1}+\ldots+v_{z_{n}^{*}}\right)$ where the $v_{i}$ are independent of 
$Z_{n}^{*}$ and are independent and identically distributed, each with the distribution of $z_{r}-m^{r}$. We can thus apply the lemma in both cases and obtain bounds which we call $c_{n}, d_{n}$ respectively. It remains to show that $\sum_{n=1}^{\infty} c_{n}<\infty, \sum_{n=1}^{\infty} d_{n}<\infty$. We shall indicate the proof for $\sum_{n=1}^{\infty} e_{n}$; that for $\sum_{n=1}^{\infty} d_{n}$ follows similarly.

What we have to demonstrate is that $\sum_{n=1}^{\infty} E\left[\left(z_{n}^{*}\right)^{-\frac{1}{2}} a_{z_{n}^{*}}\right)<\infty$, $\sum_{n=1}^{\infty} E\left(\left(Z_{n}^{*}\right)^{\frac{1}{2}} b_{Z_{n}^{*}}\right)<\infty$ and $\sum_{n=1}^{\infty} E\left(Z_{n}^{*} c_{Z_{n}^{*}}\right)<\infty$ where $a_{n}, b_{n}, c_{n}$ are defined in the lemma with $\xi_{i}$ having the distribution of $W-1$. The proofs of the convergence of these three series are identical in form. They depend on results of [4] where it is, in essence, shown in the proof of Theorem 4 that under the conditions of the theorem and if $\left\{n_{k}, k=1,2,3, \ldots\right\}$ is a sequence of integers with $n_{k} \sim K c^{2 k}$ as $k \rightarrow \infty(K>0, c>1)$, then

$$
\sum_{k=1}^{\infty} n_{k}^{-\frac{1}{2}} a_{n_{k}} \leq K_{1}<\infty, \sum_{k=1}^{\infty} n_{k}^{\frac{1}{2} b_{n}} \leq K_{2}<\infty, \sum_{k=1}^{\infty} n_{k} c_{n_{k}} \leq K_{3}<\infty
$$

for certain $K_{1}, K_{2}, K_{3}$ independent of $K$.

For $u>0$, let

$$
a_{u}=\int_{|x|<\sqrt{u}}|x|^{3} d P\left(\sigma^{-1}\left(m^{2}-m\right)^{\frac{1}{2}}(W-1) \leq x\right) .
$$

We have 


$$
\begin{aligned}
E\left(\left(Z_{n}^{*}\right)^{-\frac{1}{2}} a_{Z_{n}^{*}}\right) & =\int_{0}^{\infty}\left(x m^{n}\right)^{-\frac{1}{2}} a m^{n} d P\left(m^{-n} z_{n}^{*} \leq x\right) \\
& =\sum_{k=0}^{\infty} \int_{k \leq x<k+1}\left(x m^{n}\right)^{-\frac{1}{2} a} x^{n} d P\left(m^{-n} z_{n}^{*} \leq x\right) \\
& \leq \sum_{k=0}^{\infty} k^{-\frac{1}{2} m^{-\frac{1}{2} n} a}(k+1) m^{n^{P}}\left(k \leq m^{-n} z_{n}^{*}<k+1\right) \\
& \leq c_{1} \sum_{k=0}^{\infty}\left[(k+1) m^{n+1}\right]^{-\frac{1}{2} a}\left[(k+1) m^{n+1}\right] P\left(k \leq m^{-n} Z_{n}^{*}\right),
\end{aligned}
$$

where $c_{1}$ is a suitable positive constant and $[x]$ denotes the integer part of $x$. Then using Chebyshev's inequality, $P\left(m^{-n} z_{n}^{*} \geq k\right) \leq c k^{-2}$, and

$$
\begin{aligned}
\sum_{n=1}^{\infty} E\left(\left(z_{n}^{*}\right)^{-\frac{1}{2}} a_{Z_{n}^{*}}\right) & \leq c_{1} \sum_{n=1}^{\infty} \sum_{k=1}^{\infty}\left[k m^{n+1}\right]^{-\frac{1}{2} a}{ }_{\left[k m^{n}+1\right]} P\left(k-1 \leq m^{-n} Z_{n}^{*}\right) \\
& \leq c_{1} \sum_{n=1}^{\infty}\left[m^{n}+1\right]^{-\frac{1}{2} a}\left[m^{n}+1\right] \\
& \left.\quad+c_{1} c \sum_{k=2}^{\infty}(k-1)^{-2} \sum_{n=1}^{\infty}\left[k m^{n+1}\right]^{-\frac{1}{2}} a k^{n+1}\right] \\
& \leq c_{2}+c_{3} \sum_{k=2}^{\infty}(k-1)^{-2}<\infty,
\end{aligned}
$$

as required. That $\sum_{n=1}^{\infty} E\left(\left(Z_{n}^{*}\right)^{\frac{1}{2}} b_{Z_{n}^{*}}\right)<\infty$ and $\sum_{n=1}^{\infty} E\left(Z_{n}^{*} Z_{n}^{*}\right)<\infty$ follow in the same fashion.

Proof of Theorem 2. This follows the same lines as the proof of the theorem of [6]. We just make use of Theorem 1 and the inequality (6) instead of the results based on the Berry-Esseen inequality employed in [6]. We then obtain

$$
\lim _{n \rightarrow \infty} \frac{z_{n+r}-m^{r} z_{n}}{\left(2 \sigma_{r}^{2} v_{n}^{2} z_{n}{ }_{n} \log n\right)^{\frac{1}{2}}}=1, \quad \lim _{n \rightarrow \infty} \inf \frac{z_{n+r^{-m}} z_{n}}{\left(2 \sigma_{r}^{2} v_{n}^{2} z_{n} \log n\right)^{\frac{1}{2}}}=-1
$$

almost surely on $\{W>0\}$. The required results for $\left(z_{n+r}-m^{r} z\right)\left(2 \sigma_{r}^{2} z_{n} \log n\right)^{-\frac{1}{2}}$ then follow since $v_{n}+1$ as $n \rightarrow \infty$. We have, for example, since $z_{n} \rightarrow \infty$ almost surely on $\{W>0\}$, 


$$
\begin{aligned}
& \underset{n \rightarrow \infty}{\lim \sup } \frac{z_{n+x^{-m}} z_{n}}{\left(2 \sigma_{r}^{2} z_{n} \log n\right)^{\frac{1}{2}}} \leq \lim \sup _{n \rightarrow \infty} \frac{z_{n+x^{-m}} z_{n}}{\left(2 \sigma^{2} v_{n}^{2} z_{n}^{2} \log n\right)^{\frac{1}{2}}} \\
& \leq \lim _{n \rightarrow \infty} \sup _{n+r^{-m} z_{n}} \frac{z_{n}}{\left(2 \sigma_{n}^{2} Z_{n} \log n\right)^{\frac{1}{2}}} \lim _{n \rightarrow \infty} \sup \frac{1}{v_{Z}} \\
& =\lim _{n \rightarrow \infty} \sup \frac{z_{n+r^{-m}}{ }^{r} z_{n}}{\left(2 \sigma_{r}^{2} z_{n} \log n\right)^{\frac{1}{2}}}
\end{aligned}
$$

on $\{W>0\}$. The remainder of the proof goes through exactly as in [6]. We show

$$
\lim _{n \rightarrow \infty} \frac{\left|z_{n}-m^{n} w\right|}{\left(2 \sigma^{2}\left(m^{2}-m\right)^{-1} u_{z_{n}^{2}}^{2} z_{n} \log n\right)^{\frac{1}{2}}} \leq 1
$$

from which it follows that

$$
\lim _{n \rightarrow \infty} \frac{\left|z_{n}-m^{n} w\right|}{\left(2 \sigma^{2}\left(m^{2}-m\right)^{-1} z_{n} \log n\right)^{\frac{1}{2}}} \leq 1,
$$

and the remainder of the argument of [6] can be repeated word for word.

\section{The process with immigration}

Let $X_{0}=1, X_{1}, X_{2}, \ldots$ denote a Galton-Watson process with immigration whose offspring distribution has the distribution of $Z_{1}$ with $1<E Z_{1}=m$ and $0<\operatorname{var} Z_{1}=\sigma^{2}<\infty$. We shall also suppose that the immigration distribution has a finite mean. We refer the reader to Seneta [9] for a detailed description of the process. Under the present conditions, the theorem of [9] ensures that $m^{-n_{X}}{ }_{n}$ converges almost surely to a proper random variable $V$ with finite mean $E V$ and such that $P(V=0)=0$. We shall here obtain the following theorems which extend results presented in Heyde and Seneta [8].

THEOREM 3. Let $1<m=E Z_{1}$ and $0<\operatorname{var} Z_{1}=\sigma^{2}<\infty$. Then 


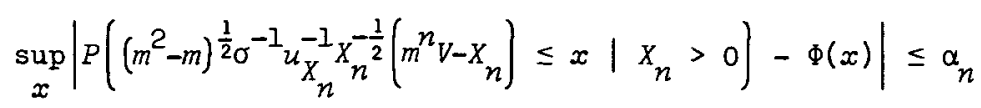

and

$$
\sup _{x}\left|P\left(\sigma_{r}^{-1} v_{X}^{-1} X_{n}^{-\frac{1}{2}}\left(X_{n+r}-m^{r} X_{n}\right) \leq x \mid X_{n}>0\right)-\Phi(x)\right| \leq \beta_{n},
$$

where $\left\{\alpha_{n}\right\},\left\{\beta_{n}\right\}$ are certain sequences of positive constants satisfying $\sum_{n=1}^{\infty} \alpha_{n}<\infty$ and $\sum_{n=1}^{\infty} \beta_{n}<\infty$. Here $\sigma_{r}, u_{n}, v_{n}$ are as defined in the statement of Theorem 1 .

Explicit forms for $\alpha_{n}$ and $\beta_{n}$ can be found by applying the lemma.

THEOREM 4. Suppose that $1<m=E Z_{1}$ and $0<\operatorname{var} Z_{1}=\sigma^{2}<\infty$. Then, with probability one,

$$
\begin{aligned}
& \lim _{n \rightarrow \infty} \frac{x_{n+r^{-m}} m_{n}}{\left(2 \sigma_{r}^{2} X_{n} \log n\right)^{\frac{1}{2}}}=1, \quad \lim _{n \rightarrow \infty} \inf \frac{X_{n+r^{-}} m^{r} X_{n}}{\left(2 \sigma_{r}^{2} X_{n} \log n\right)^{\frac{1}{2}}}=-1, \\
& \lim _{n \rightarrow \infty} \frac{m^{n} V-X_{n}}{\left(2 \sigma^{2}\left(m^{2}-m\right)^{-1} X_{n} \log n\right)^{\frac{1}{2}}}=1, \quad \lim _{n \rightarrow \infty} \inf \frac{m^{n} V-X n}{\left(2 \sigma^{2}\left(m^{2}-m\right)^{-1} X_{n} \log n\right)^{\frac{1}{2}}}=-1 \text {, }
\end{aligned}
$$

where $r$ is any fixed positive integer.

Theorems 3 and 4 extend the scope of results given in Theorems 2 and 3 of [8] under the additional condition that $E Z_{1}^{3}<\infty$. The form of the bounds obtained in the present Theorem 3 is however, of necessity, much more complicated in the more general case. The present Theorem 4 preserves exactly the form of Theorem 3 of [8] under the more general conditions.

The proofs of Theorems 2 and 3 follow the same lines as those of Theorems 1 and 2, again using the lemma and (6). We make use of the representations

$$
m^{n} V-X_{n}=\left(w^{(1)}-1\right)+\ldots+\left(W^{\left(X_{n}\right)}-1\right)+I^{(n)} \text { a.s. }
$$

and 


$$
X_{n+r}-m^{r} X_{n}=\left(z_{r}^{(1)}-m^{r}\right)+\ldots+\left(z_{r}^{\left(X_{n}\right)}-m^{r}\right)+y_{r, n}
$$

of [8] and the points noted in the proof of Theorem 3 of [8]. The only real point of difference in the proofs involves showing that we can choose a sequence $\left\{\varepsilon_{n}\right\}$ with $\varepsilon_{n} \rightarrow 0$ as $n \rightarrow \infty$ such that $\sum_{n=1}^{\infty} \varepsilon_{n}<\infty$ and $\sum_{n=1}^{\infty} \varepsilon_{n}^{-1} E\left(X_{n}^{-\frac{1}{2}} w_{X}^{-1} \mid X_{n}>0\right)<\infty$ where $w_{n}$ is either $u_{n}$ or $v_{n}$. We know that $u_{n} \uparrow I, v_{n} \uparrow 1$ so that $w_{n} \uparrow 1$ as $n \rightarrow \infty$. Thus, conditional on $x_{n}>0, w_{X_{n}} \geq w_{1}$ and hence

$$
\begin{aligned}
\sum_{n=1}^{\infty} \varepsilon_{n}^{-1} E\left(x_{n}^{-\frac{1}{2}} w_{X_{n}}^{-1} \mid x_{n}>0\right) & \leq w_{1}^{-1} \sum_{n=1}^{\infty} \varepsilon_{n}^{-1} E\left(x_{n}^{-\frac{1}{2}} \mid x_{n}>0\right) \\
& \leq w_{1}^{-1} \sum_{n=1}^{\infty} \varepsilon_{n}^{-1}\left(E\left(x_{n}^{-1} \mid x_{n}>0\right)\right)^{\frac{1}{2}} .
\end{aligned}
$$

Now, using Lemma 2.3 of [8] we have $\varepsilon_{n}=\theta^{n}$ with $0<\gamma<\theta<1$,

$$
\sum_{n=1}^{\infty} \varepsilon_{n}^{-1}\left(E\left(X_{n}^{-1} \mid x_{n}>0\right)\right)^{\frac{1}{2}} \leq \sum_{n=1}^{\infty} \theta^{-n} \gamma^{n}<\infty .
$$

Thus, with this choice of $\varepsilon_{n}, \sum_{n=1}^{\infty} \varepsilon_{n}<\infty$ and $\sum_{n=I}^{\infty} \varepsilon_{n}^{-1} E\left(X_{n}^{-\frac{1}{2}} w_{X}^{-1} \mid X_{n}>0\right)<\infty$, as required.

\section{References}

[1] Wolfgang J. Bühler, "Ein zentraler Grenzwertsatz für Verzweigungsprozesse", 2. Wahrscheinlichkeits theorie verw. Gebiete 11 (1969), 139-141. 
[2] Theodore E. Harris, The theory of branching processes (Die Grundlehren der Mathematischen Wissenschaften in Einzeldarstellungen, Band 119. Springer-Verlag, Berlin, Göttingen, Heidelberg, 1963; Prentice-Hall, Englewood Cliffs, New Jersey, 1963).

[3] C.C. Heyde, "On the influence of moments on the rate of convergence to the normal distribution", 2. Wahrscheinlichkeits theorie verw. Gebiete 8 (1967), 12-18.

[4] C.C. Heyde, "Some properties of metrics in a study on convergence to normality", Z. Wahrscheinlichkeits theorie verw. Gebiete 11 (1969), 181-192.

[5] C.C. Heyde, "Some central limit analogues for super-critical Galton-Watson processes", J. Appl. Probability 8 (1971), 52-59.

[6] C.C. Heyde, "Some almost sure convergence theorems for branching processes", Z. Wahrscheinlichkeitstheorie verw. Gebiete (to appear).

[7] C.C. Heyde and 8.M. Brown, "An invariance principle and some convergence rate results for branching processes", $z$. Wahrscheinlichkeitstheorie verw. Gebiete (to appear).

[8] C.C. Heyde and E. Seneta, "Analogues of classical limit theorems for the supercritical Galton-Watson process with immigration", Math. Biosci. (to appear).

[9] E. Seneta, "A note on the supercritical Galton-Watson process with immigration", Math. Biosci. 6 (1970), 305-312.

Department of Statistics,

School of General Studies,

Australian National University,

Canberra, ACT. 